\section{RMD Open}

Rheumatic \&

Musculoskeletal Diseases

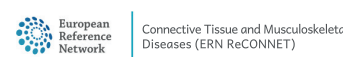

\title{
IgG4-related diseases: state of the art on clinical practice guidelines
}

Luca laccarino, ${ }^{1}$ Rosaria Talarico, ${ }^{2}$ Carlo Alberto Scirè, ${ }^{3}$ Zahir Amoura, ${ }^{4}$ Gerd Burmester, ${ }^{5}$ Andrea Doria, ${ }^{1}$ Karim Faiz, ${ }^{6}$ Charissa Frank, ${ }^{7}$ Eric Hachulla, ${ }^{8}$ Miguel Hie, ${ }^{4}$ David Launay, ${ }^{8}$ Carlomaurizio Montecucco, ${ }^{9}$ Sara Monti, ${ }^{9}$ Luc Mouthon, ${ }^{10}$ Angela Tincani, ${ }^{11}$ Paola Toniati, ${ }^{11}$ Pieter Martin Van Hagen, ${ }^{6}$ Ronald F Van Vollenhoven, ${ }^{12}$ Stefano Bombardieri, ${ }^{13}$ Ulf Mueller-Ladner, ${ }^{14}$ Matthias Schneider, ${ }^{15}$ Vanessa Smith, ${ }^{16,17}$ Maurizio Cutolo, ${ }^{18}$ Marta Mosca, ${ }^{2,19}$ Tobias Alexander ${ }^{5}$

To cite: laccarino $\mathrm{L}$, Talarico R, Scirè CA, et al. IgG4-related diseases: state of the art on clinical practice guidelines. RMD Open 2019;4:e000787. doi:10.1136/ rmdopen-2018-000787

- Prepublication history for this paper is available online. To view these files, please visit the journal online (http://dx.doi. org/10.1136/rmdopen-2018000787).

Received 5 August 2018 Revised 24 November 2018 Accepted 3 December 2018

Check for updates

(C) Author(s) (or their employer(s)) 2019. Re-use permitted under CC BY-NC. No commercial re-use. See rights and permissions. Published by BMJ.

For numbered affiliations see end of article.

Correspondence to Dr Tobias Alexander; Tobias.Alexander@charite.de

\section{ABSTRACT}

Immunoglobulin G4-related diseases (IgG4-RD) are a group of chronic relapsing-remitting inflammatory conditions, characterised by tissue infiltration with lymphocytes and IgG4-secreting plasma cells, fibrosis and a usually favourable response to steroids.

In this narrative review, we summarise the results of a systematic literature research, which was performed as part of the European Reference Network ReCONNET, aimed at evaluating existing clinical practice guidelines (CPGs) and recommendations in IgG4-RD. From 167 publications initially obtained from a systematic literature search, only one was identified as a systematic multispecialist, evidence-based, consensus guidance statement on diagnosis and treatment of IgG4-RD, which may be recommended for use as CPG in IgG4-RD.

With the recognition of a limited evidence based in this increasingly recognised disease, the group discussion has identified the following unmet needs: lack of shared classification criteria, absence of formal guidelines on diagnosis, no evidence-based therapeutic recommendations and lack of activity and damage indices. Areas of unmet needs include the difficulties in diagnosis, management and monitoring and the scarcity of expert centres.

\section{INTRODUCTION}

Immunoglobulin G4-related disease (IgG4$\mathrm{RD})$ is an increasingly recognised immunemediated chronic relapsing-remitting inflammatory condition, characterised by tissue infiltration with lymphocytes and IgG4-secreting plasma cells, fibrosis and a usually favourable response to steroids. The commonly shared features include tumour-like swelling of involved organs, which in the majority of patients is indolent, particularly in early stages of disease. Pain is usually a consequence of the obstruction or compression due to the presence of mass lesions and their complications. Multiorgan involvement may either be

\section{Key messages}

What is already known about this subject?

- Immunoglobulin G4-related disease (IgG4-RD) is a relatively new defined clinical entity with lack of evidence base to guide clinical care.

- There is an unmet need for clinical practice guidelines for diagnosis, classification and standard of care in patients with IgG4-RD.

What does this study add?

- This systematic review reports the state of the art on existing clinical guidelines and unmet needs in the management of patients with IgG4-RD.

How might this impact on clinical practice?

- The European Reference Network on Rare and Complex Connective Tissue and Musculoskeletal Diseases (ReCONNET) will provide better and safer healthcare in IgG4-RD by promoting validated standard clinical guidelines.

present at the same time (synchronously) or different sites can be affected at various time periods (metachronously). Major presentations of IgG4-RD include type 1 autoimmune pancreatitis, salivary gland disease, orbital disease and retroperitoneal fibrosis. Irrespective of which organ or tissue is involved, the hallmarks of IgG4-RD are lymphoplasmacytic infiltrates rich in IgG4-positive plasma cells, storiform fibrosis and obliterative phlebitis. The clinical suspicion may arise from elevated plasma IgG4 levels, but the definitive diagnosis is based on histology and other cell-based diagnostics as serum concentration may be within the normal range. To prevent fibrosis progression and organ destruction, a treatment of induction and maintenance of remission is necessary. The recommended first-line agents are glucocorticoids. However, 
relapses are frequent during tapering, so glucocorticoid-sparing immunosuppressive agents are usually considered, although adequate controlled studies on their efficacy are lacking. Finally, surgery or radiotherapy may be necessary in case of serious organ damage.

In this narrative review, we summarise the results of a systematic literature research, which was performed as part of the ERN ReCONNET project, dedicated to evaluation of currently available clinical practice guidelines (CPGs) or recommendations. Subsequently, clinicians' and patients' unmet needs of IgG4RD were discussed.

\section{METHODS}

\section{ReCONNET network}

ERN ReCONNET (Rare CONnective tissue and musculoskeletal diseases NETwork) is a European Reference Network on Rare and Complex Connective Tissue and Musculoskeletal Diseases funded by the European Union's Health Program to promote better and safer healthcare, define proper organisational assessment and identify standard and cost-effective pathways for the management of rare and complex connective tissue diseases. The Network includes rheumatologists, internists and immunologists from 26 selected centres in eight different countries across Europe (http://reconnet. ern-net.eu). One of the first network targets was evaluate currently available CPGs or recommendations in a systematic literature search to identify potential unmet needs in the most relevant rare autoimmune diseases.

\section{Systematic literature search}

We carried out a systematic search in PubMed and EMBASE based on controlled terms (MeSH and Emtree) and keywords of the disease and publication type (CPGs). We reviewed all published articles in order to identify existing CPGs on diagnosis, monitoring and treatment, according to the Institute of Medicine 2011 definition (CPGs are statements that include recommendations intended to optimise patient care that are informed by a systematic review of evidence and an assessment of the benefits and harms of alternative care options).

The disease coordinators (DCs) of the ERN ReCONNET for IgG4-RD had assigned the work on CPGs to the healthcare providers (HCPs) involved. Moreover, in order to implement the list of guidelines provided by PubMed and EMBASE search, the group performed also a hand search. A first screening among papers included in the final list (systematic search+hand search) based on title and abstract selected evidence-based medicine guidelines. A general assessment of the CPGs had been performed following the AGREE II tool checklist not for formal appraisal but only to inform discussion. A discussion group composed of DCs (LI and TA) and disease representative (DR) was set for the evaluation of the existing CPGs and to identify the unmet needs.

More precisely, the following search strategy was used to identify publications from the databases:
Medline (PubMed)

(IgG4-related [All Fields] AND ("disease"[MeSH Terms] OR "disease" [All Fields])) AND ("Practice Guideline"[Publication Type] OR "Practice Guidelines As Topic" [MeSH Terms] OR Practice Guideline[Publication Type] OR "Practice Guideline"[Text Word] OR "Practice Guidelines"[Text Word] OR "Guideline"[Publication Type] OR "Guidelines As Topic" [MeSH Terms] OR Guideline[Publication Type] OR "Guideline"[Text Word] OR "Guidelines" [Text Word] OR "Consensus Development Conference"[Publication Type] OR "Consensus Development Conferences As Topic" [MeSH Terms] OR "Consensus" [MeSH Terms] OR "Consensus"[Text Word] OR "Recommendation"[Text Word] OR "Recommendations" [Text Word] OR "Best Practice" [Text Word] OR "Best Practices" [Text Word]).

\section{EMBASE}

('immunoglobulin g4 related disease'/exp OR 'igg4 related disease' OR 'immunoglobulin $\mathrm{g} 4$ related disease') AND ('practice guideline'/exp OR 'practice guideline' OR 'practice guidelines'/exp OR 'practice guidelines' OR 'clinical practice guideline' / exp OR 'clinical practice guideline' OR 'clinical practice guidelines'/exp OR 'clinical practice guidelines' OR 'clinical practice guidelines as topic'/exp OR 'clinical practice guidelines as topic' OR 'guideline' / exp OR 'guideline' OR 'guidelines' / exp OR 'guidelines' OR 'guidelines as topic' / $\exp$ OR 'guidelines as topic' OR 'consensus development'/exp OR 'consensus development' OR 'consensus development conference'/exp OR 'consensus development conference' OR 'consensus development conferences' / exp OR 'consensus development conferences' OR 'consensus development conferences as topic'/exp OR 'consensus development conferences as topic' OR 'consensus' / exp OR 'consensus' OR 'recommendation' OR 'recommendations') AND [embase]/lim NOT [medline]/lim.

\section{STATE OF THE ART ON CPGS}

\section{Identification of existing CPGs}

The systematic literature search initially identified a total of 165 citations by the ERN ReCONNET central team (figure 1). After performing title and abstract evaluation by disease representatives, eight papers were selected for further review. After full-text review by the disease coordinators, four CPGs were finally selected for further evaluation using full-text assessment, ${ }^{1-4}$ which were subsequently endorsed by the disease representatives.

\section{CPG characteristics}

The general characteristics of the four CPGs are summarised in table 1. Of the four preselected publications, only one was identified as a systematic multispecialist, evidencebased, consensus guidance statement on diagnosis and treatment of IgG4-RD, which may be recommended for use as CPG. ${ }^{1}$ This publication was an International Consensus Guidance Statement on the management and treatment of IgG4-RD, resulting from an expert panel 


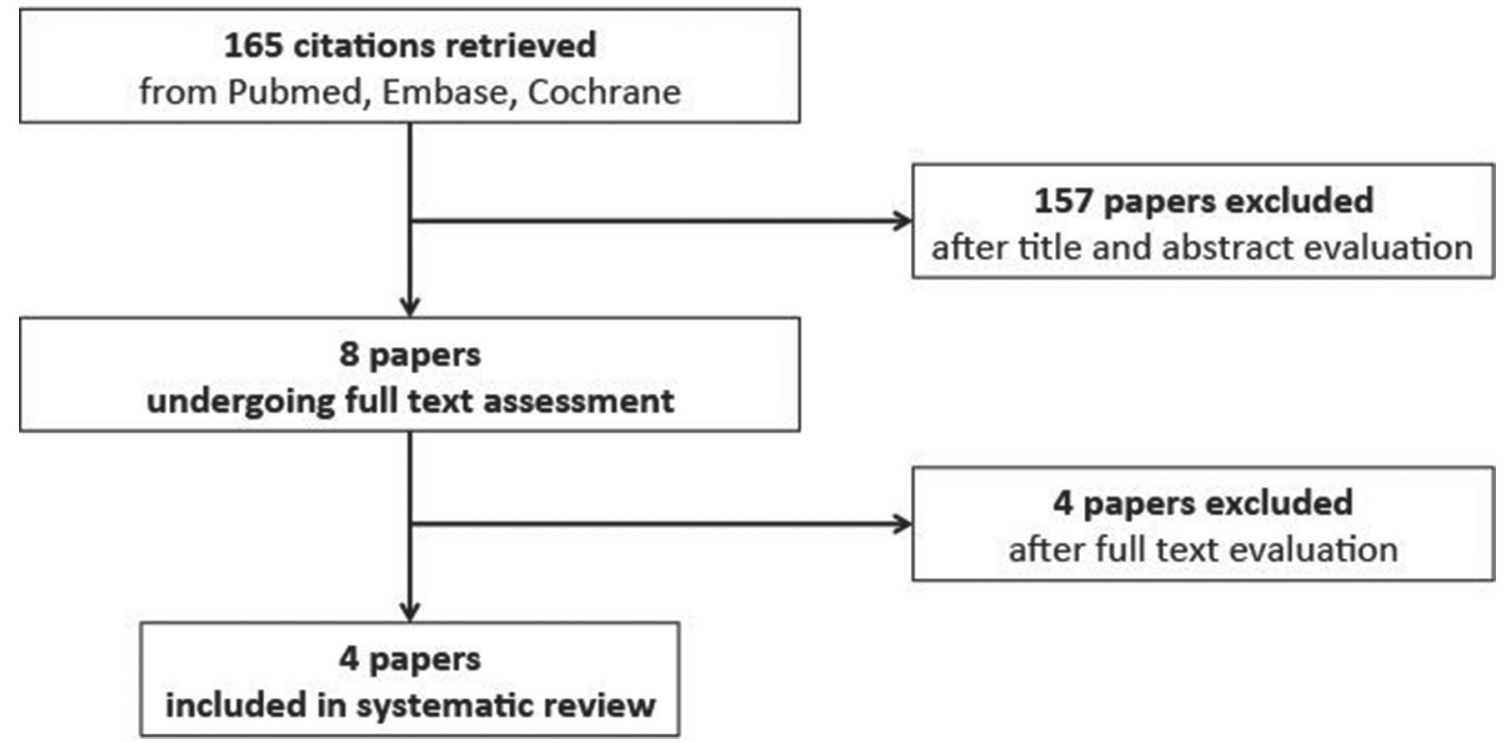

Figure 1 Stepwise search strategy for selection and evaluation of published clinical practice guidelines for lgG4-RD.

of 42 IgG4-RD experts from eight medical specialties (18 gastroenterologists, 13 rheumatologists and 11 other specialists and subspecialists). Although these guidelines are relevant for clinical practice, the evidence level reported in the paper varies between IIb and IV, given the lack of solid and endorsed classification criteria and randomised controlled trials (RCTs) in this field. ${ }^{1}$ As a result, there was broad consensus that

- The most accurate assessment of IgG4-RD is based on a full clinical history, physical examination, selected laboratory investigations and appropriate radiology studies.

- Diagnostic confirmation by biopsy is strongly recommended for the exclusion of malignancies and other IgG4-RD mimics.

- All patients with symptomatic, active IgG4-RD and a subset of patients with asymptomatic disease require treatment, some urgently.

- Glucocorticoids are the first-line agent for remission induction unless contraindications are present.
- Some but not all patients require the combination of glucocorticoids and a steroid-sparing immunosuppressive agent from the start of treatment.

- Following a successful course of induction therapy, certain patients benefit from maintenance therapy.

- Re-treatment with glucocorticoids is indicated in patients who relapse off of treatment following successful remission induction. Following relapse, the introduction of a steroid-sparing agent for continuation in the remission maintenance period should be considered.

The remaining three selected GPGs were only reviews or consensus statements on existing data for terminology, pathology and immunopathology of IgG4-RD. ${ }^{2-4}$ They lack accepted methods for systematic review and represent rather expert consensus than real guidelines or recommendation with suboptimal clinical practice use, which may not be recommended as a guideline for use (table 1).

Table 1 Summary of the evaluation of existing guidelines

\begin{tabular}{ll}
\hline Author and year & Description \\
\hline Khosroshahi et al, 2015 & $\begin{array}{l}\text { Systematic review, multiple specialists, guidelines for diagnostic and treatment, } 1 \\
\text { so far first real guidelines; very helpful for the physician in clinical practice }\end{array}$ \\
\hline Deshpande et al, 2013 & $\begin{array}{l}\text { Review on existing data for terminology, pathology and immunopathology, no } \\
\text { real guidelines, no systematic review; recommendations are lacking, so the } \\
\text { usefulness of this paper is limited }\end{array}$ \\
Deshpande et al, 2012 & $\begin{array}{l}\text { Consensus statement on pathology, only expert opinion, no clear systematic } \\
\text { review; no systematic methods for formulating recommendations key } \\
\text { histopathological features and lgG4 assessment are clearly defined }\end{array}$ \\
& $\begin{array}{l}\text { Review on terminology based on expert opinion. Although experts from } \\
\text { different specialties were present, this is rather expert consensus than a clear } \\
\text { recommendation; no systematic guidelines are reported and key messages are } \\
\text { not easily identifiable }\end{array}$ \\
\hline
\end{tabular}




\section{UNMET NEEDS}

Clinicians' unmet needs

The following unmet needs have been identified and discussed by the DCs and DRs:

\section{Lack of shared classification criteria}

There is no international consensus on classification criteria for IgG4-RD. Therefore, development of validated classification criteria should be the first step to guide clinicians in stratifying patients and to provide the basis for further collaborative and comparative research studies in the field. In this sense, the European League Against Rheumatism (EULAR) and American College of Rheumatology (ACR) are currently jointly supporting a Task Force for the development of classification criteria for IgG4-RD that will hopefully be available in the near future.

\section{No formal guidelines for diagnosis available}

Diagnosis of IgG4-RD is challenging, given the heterogeneity of clinical symptoms and laboratory results. The prevalence of IgG4-RD is probably underestimated, especially in Europe and North America, where the awareness of its existence may be lower than in Asian countries, where the disease was initially described. Although serum IgG4 concentrations may be helpful, they have certainly lost importance in the diagnosis. ${ }^{5}$ Histology is still the gold standard for diagnosis, but evidence-based criteria in this field are scarce. In the last years, several studies have been published on possible diagnostic markers and predictive factors. For example, assessment of plasmablast levels by flow cytometry has recently emerged as a promising new diagnostic modality. ${ }^{6}$ However, more evidence in this field is necessary to provide the basis for standardised algorithms in the diagnostic process. Further studies are required that need to be performed in an international collaborative setting, including all involved disease specialists, that is, rheumatology, gastroenterology, vascular medicine, surgery, radiology, pathology and clinical immunology.

\section{Indices for disease activity and damage are lacking}

Similar to other inflammatory rheumatic diseases, IgG4-RD presents with relapsing-remitting courses that may eventually result in tissue damage. Therefore, standardised indices for disease activity and damage need to be developed and validated. In addition, the place and relevance of imaging techniques (eg, ultrasound, CT, MRI and PET scans) in diagnosis and follow-up of the disease should be evaluated.

\section{Therapy is not evidence based}

Although IgG4-RD frequently represents an indolent condition, treatment is usually required to reduce chronic inflammation and to prevent progressive tissue fibrosis. Furthermore, untreated IgG4-RD with elevated inflammatory markers may lead to secondary AA amyloidosis. ${ }^{78}$ Therefore, symptoms and risk of irreversible organ damage due to fibrosis and also modulation of the underlying chronic inflammatory condition should be taken into account for treatment decisions. Single-centre observations suggest favourable responses to glucocorticoids, which are regarded as first-line therapy, but relapses are frequently observed during steroid tapering. Disease-modifying drugs (DMARDs) have proven efficacy in some cases, but large RCTs investigating the outcome of various DMARDs are lacking. A recent nationwide retrospective study showed that rituximab is effective for both induction therapy and relapse treatment in IgG4RD. ${ }^{9}$ Hence, RCTs are urgently required in the field, but difficult to conduct given the rarity and heterogeneity of the disease. Alternatively, evidence from non-randomised or uncontrolled studies may also be used for further developments. In addition, validated tools for treatment response are lacking and no treat-to-target criteria are available. Recently, an IgG4-RD responder index ${ }^{10}$ has been developed and an international validation study is ongoing. To date, this index is the only available instrument to assess IgG4-RD and its use needs to be encouraged in clinical practice.

\section{Patient unmet needs}

IgG4-RD represents a relatively new disease entity and awareness of the disease among healthcare providers is still inadequate and limited to few disease experts. Subsequently, the lag time from symptom onset to diagnosis is still unacceptably high and may be associated with accrual of irreversible organ damage before specific treatments are provided.

Each patient is different in relation to their symptoms as well as the approach of their care. Most care is also not evidence based, which may be an additional burden for the patient as some healthcare systems only support evidence-based care. Therefore, patients with IgG4-RD require special attention, especially with respect to interdisciplinary dialogue with treating physicians, individual support from patient representatives as well as guidance to address the psychosocial impact of the disease.

Centres of expertise for IgG4-RD are currently limited and not easy to identify by patients. In addition, the patient support system is still insufficient, as there are almost no disease-specific patient organisations established at the moment on national and international level.

In conclusion, much effort is needed at multiple levels to meet the specific demands of patients suffering from IgG4-RD in providing optimised patient care, which may hopefully be accomplished in the framework of the ERN ReCONNET.

\section{CONCLUSIONS}

IgG4-RD is a relatively new defined clinical entity. Therefore, formal disease classification and guidelines for diagnosis and treatment are still widely lacking. Given the rarity and heterogeneity of this disease, RCT may only be accomplished in international multicentre trials with support of pharmaceutical companies. The current 
development of disease classification criteria by the EULAR and ACR task force will be the first step to harmonise terminology and to provide the basis for further collaborative studies on diagnostic recommendations and treatment guidelines, which need to be endorsed by scientific international societies (ie, EULAR, ACR). In the absence of RCTs, the implementation of non-randomised pilot studies will help to increase the level of evidence of therapeutic guidelines.

\section{Author affiliations}

${ }^{1}$ Rheumatology Unit, Department of Medicine, A0 Padova and University of Padua, Padua, Italy

${ }^{2}$ Rheumatology Unit, AOU Pisana, Pisa, Italy

${ }^{3}$ Section of Rheumatology, Department of Medical Sciences, University of Ferrara, Ferrara, Italy

${ }^{4}$ Department of Internal Medicine, Hospital Pitié-Salpêtrière, Assistance PubliqueHôpitaux de Paris, Paris, France

${ }^{5}$ Department of Rheumatology and Clinical Immunology, Charité-University Medicine Berlin, Berlin, Germany

${ }^{6}$ Department of Internal Medicine and Immunology, Erasmus MC, Rotterdam, The Netherlands

${ }^{7}$ Bindweefsel.be, Koersel, Belgium

${ }^{8}$ Département de Médecine Interne et Immunologie Clinique, Centre de Référence des Maladies Systémiques et Auto-Immunes Rares du Nord-Ouest (CERAINO), LIRIC, INSERM, Univ. Lille, CHU Lille, Lille, France

${ }^{9}$ Division of Rheumatology, University and IRCCS Policlinico S Matteo Foundation, Pavia, Italy

${ }^{10}$ Service de Médecine Interne, Hôpital Cochin, Centre de Référence Maladies systémiques Autoimmunes Rares d'lle de France, Assistance Publique-Hôpitaux de Paris (AP-HP), Université Paris Descartes, Paris, France

${ }^{11}$ Rheumatology and Clinical Immunology Unit, Civil Hospital, Brescia, Italy

${ }^{12}$ Clinical Immunology and Rheumatology, Amsterdam Rheumatology and Immunology Center, Academic Medical Center/University of Amsterdam, Amsterdam, The Netherlands

${ }^{13}$ University of Pisa, Pisa, Italy

${ }^{14}$ Department of Rheumatology and Clinical Immunology, Kerckhoff Klinik, JustusLiebig University of Giessen, Bad Nauheim, Germany

${ }^{15}$ Department of Rheumatology, Universitätsklinikum Düsseldorf, Düsseldorf, Germany

${ }^{16}$ Department of Rheumatology, Ghent University Hospital, Ghent, Belgium

${ }^{17}$ Department of Internal Medicine, Ghent University, Ghent, Belgium

${ }^{18}$ Academic Division of Clinical Rheumatology, Department of Internal Medicine, IRCCS Polyclinic Hospital San Martino, University of Genoa, Genoa, Italy

${ }^{19}$ Rheumatology Unit, University of Pisa, Pisa, Italy

Acknowledgements We thank all members of the Steering Committee of the ERN ReCONNET for their huge commitment during this work.

Contributors LI, RT, CAS, MM and AT: substantial contributions to the conception and design of the work; the acquisition, analysis and interpretation of data; drafting the work and revising it critically for important intellectual content; final approval of the version to be published; agreement to be accountable for all aspects of the work in ensuring that questions related to the accuracy or integrity of any part of the work are appropriately investigated and resolved. ZA, GB, AD, KF, CF, EH, MH DL, CM, SM, LM, AT, PT, PMVH, RVV: substantial contributions to the analysis and interpretation of data; final approval of the version to be published; agreement to be accountable for all aspects of the work in ensuring that questions related to the accuracy or integrity of any part of the work are appropriately investigated and resolved. SB, UM-L, MS, VS, MC: revising the work critically for important intellectual content; final approval of the version to be published; agreement to be accountable for all aspects of the work in ensuring that questions related to the accuracy or integrity of any part of the work are appropriately investigated and resolved.

Funding This publication was funded by the European Union's Health Programme (2014-2020).

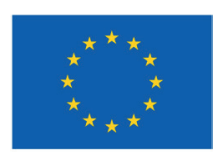

Disclaimer ERN ReCONNET is one of the 24 European Reference Networks (ERNs) approved by the ERN Board of Member States. The ERNs are co-funded by the European Commission. The content of this publication represents the views of the authors only and it is their sole responsibility; it cannot be considered to reflect the views of the European Commission and/or the Consumers, Health, Agriculture and Food Executive Agency (CHAFEA) or any other body of the European Union. The European Commission and the Agency do not accept any responsibility for use that may be made of the information it contains. Competing interests None declared.

Patient consent for publication Not required.

Provenance and peer review Commissioned; externally peer reviewed.

Data sharing statement No additional data are available.

Open access This is an open access article distributed in accordance with the Creative Commons Attribution Non Commercial (CC BY-NC 4.0) license, which permits others to distribute, remix, adapt, build upon this work non-commercially, and license their derivative works on different terms, provided the original work is properly cited, appropriate credit is given, any changes made indicated, and the use is non-commercial. See: http://creativecommons.org/licenses/by-nc/4.0

\section{REFERENCES}

1. Khosroshahi A, Wallace ZS, Crowe JL, et al. International consensus guidance statement on the management and treatment of IgG4related disease. Arthritis Rheumatol 2015;67:1688-99.

2. Deshpande V, Khosroshahi A. Diagnostic guidelines for IgG4related disease with a focus on histopathological criteria. Diagnostic Histopathology 2013;19:119-27.

3. Deshpande V, Zen Y, Chan JKC, et al. Consensus statement on the pathology of IgG4-related disease. Modern Pathology 2012;25:1181-92.

4. Stone JH, Khosroshahi A, Deshpande V, et al. Recommendations for the nomenclature of IgG4-related disease and its individual organ system manifestations. Arthritis Rheum 2012;64:3061-7.

5. Wallace ZS, Mattoo $\mathrm{H}$, Carruthers $\mathrm{M}$, et al. Plasmablasts as a biomarker for IgG4-related disease, independent of serum IgG4 concentrations. Ann Rheum Dis 2015;74:190-5.

6. Heeringa JJ, Karim AF, van Laar JAM, et al. Expansion of blood IgG4+ B, TH2, and regulatory T cells in patients with IgG4-related disease. J Allergy Clin Immunol 2018;141:1831-43.

7. Karim F, Clahsen-van Groningen M, van Laar JA. AA Amyloidosis and IgG4-related disease. N Engl J Med 2017;376:599-600.

8. Karim AF, Eurelings LEM, van Hagen PM, et al. Implications of elevated C-reactive protein and serum amyloid A levels in IgG4related disease: comment on the article by Perugino et al. Arthritis Rheumatol 2018;70:317-8.

9. Ebbo M, Grados A, Samson M, et al. Long-term efficacy and safety of rituximab in IgG4-related disease: data from a French nationwide study of thirty-three patients. Plos One 2017;12:e0183844.

10. Carruthers MN, Stone JH, Deshpande V, et al. Development of an IgG4-RD responder Index. Int J Rheumatol 2012;2012:1-7. 